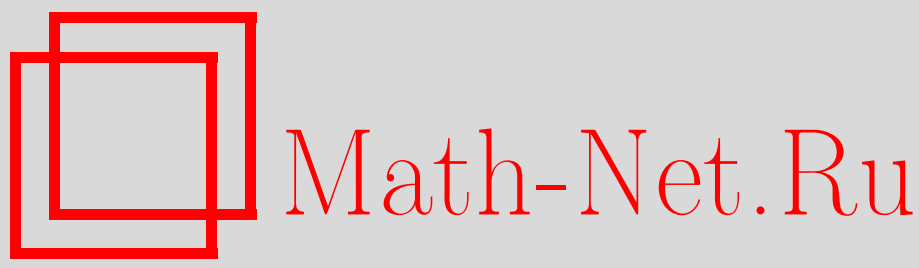

С. И. Агафонов, Е. В. Ферапонтов, Системы законов сохранения с точки зрения проективной теории конгруэнций, Изв. РАН. Сер. матем., 1996, том 60, выпуск $6,3-30$

DOI: https://doi.org/10.4213/im93

Использование Общероссийского математического портала Math-Net.Ru подразумевает, что вы прочитали и согласны с пользовательским соглашением http://www . mathnet.ru/rus/agreement

Параметры загрузки:

IP : 34.239 .49 .27

26 апреля 2023 г., 13:13:45 
УДК 517.95

С.И. Агафонов, Е.В. Ферапонтов

\section{Системы законов сохранения с точки зрения проективной теории конгруэнций}

Системе $n$ законов сохранения

$$
u_{t}^{i}=f^{i}(u)_{x}, \quad i=1, \ldots, n,
$$

ставится в соответствие $n$-параметрическое семейство прямых в $(n+1)$-мерном пространстве $A^{n+1}$, заданное уравнениями

$$
y^{i}=u^{i} y^{0}-f^{i}(u), \quad i=1, \ldots, n .
$$

Устанавливаются следующие свойства введенного соответствия:

- преобразованиям по решению системы законов сохранения отвечают проективные преобразования пространства $A^{n+1}$;

- кривым разрежения системы законов сохранения отвечают развертывающиеся поверхности семейства прямых;

- системам законов сохранения класса Темпля отвечают семейства прямых, развертывающиеся поверхности которых являются либо плоскими, либо коническими.

На языке теории конгруэнций дается описание систем законов сохранения класса Темпля в случае $n=2$.

Библиограбия: 19 наименований.

\section{§1. Введение}

Системы законов сохранения

$$
u_{t}^{i}=f^{i}(u)_{x}, \quad i=1, \ldots, n,
$$

являются одними из наиболее простых и хорошо изученных моделей нелинейных волновых процессов, для которых удается эфффективно развить теорию слабых решений (см., например, [1]-[3]). В настоящей работе строится проективно-геометрическая теория систем законов сохранения.

Для удобства читателя в $§ 1$ мы начинаем со стандартных определений кривых разрежения и ударной адиабаты. Условие их совпадения выделяет специальный класс систем законов сохранения, который был подробно изучен Темплем в [3]. Мы приводим основную теорему работы [3], дающую описание систем этого класса (теорема 1). В качестве примеров рассмотрены уравнения хроматографии и система из трех законов сохранения, возникающая в двумерной топологической теории поля. 
В $§ 2$ вводится важный класс преобразований систем законов сохранения - так называемые преобразования по решению. Устанавливается инвариантность кривых разрежения, ударной адиабаты и систем класса Темпля относительно преобразований по решению (теоремы 2 и 3 ).

В $\S 3$ рассматриваются системы из двух законов сохранения

$$
\begin{aligned}
& u_{t}^{1}=f^{1}(u)_{x} \\
& u_{t}^{2}=f^{2}(u)_{x}
\end{aligned}
$$

и указывается явный вид правых частей $f^{1}, f^{2}$, гарантируюший принадлежность системы классу Темпля.

Центральным местом работы является $\S 4$, где системе законов сохранения ставится в соответствие $n$-параметрическое семейство прямых в $(n+1)$-мерном пространстве $A^{n+1}$, заданное уравнениями

$$
\begin{aligned}
& y^{1}=u^{1} y^{0}-f^{1}(u), \\
& y^{n}=u^{n} y^{0}-f^{n}(u) .
\end{aligned}
$$

В случае $n=2$ мы получаем двухпараметрическое семейство, или, как говорят, конгруэнцию прямых в $A^{3}$. Начиная с работ Монжа, Плюккера и Куммера теория конгруэнщий является одной из наиболее красивых и ярких глав классической дифференциальной геометрии (см., например, [14]).

Установлены следующие свойства введенного соответствия:

- преобразованиям по решению системы законов сохранения отвечают проективные преобразования пространства $A^{n+1}$ (теорема 5);

- кривым разрежения системы законов сохранения отвечают развертывающиеся поверхности семейства прямых (теорема 6);

- ударной адиабате с вершиной в точке $u_{0}$ отвечают прямые семейства, которые пересекают данную прямую $y^{i}=u_{0}^{i} y^{0}-f^{1}\left(u_{0}\right)$ (теорема 7 );

- коммутирующим системам законов сохранения отвечают семейства прямых, у которых фокальные сети на соответствующих фокальных гиперповерхностях попарно находятся в соответствии Петерсона (теорема 8).

Неожиданный на первый взгляд вывод состоит в том, что теория систем законов сохранения есть не что иное, как проективная геометрия $n$-параметрических семейств прямых в $A^{n+1}$. При этом теоремы 2 и 3 , устанавливающие инвариантность кривых разрежения и ударной адиабаты относительно преобразований по решению, превращаются в элементарные факты проективной геометрии.

В $\S 5$ показано, что системам законов сохранения класса Темпля отвечают семейства прямых, развертываюшиеся поверхности которых являются либо плоскими, либо коническими (теоремы 9 и 10). На основе прозрачных геометрических рассуждений продемонстрировано, каким образом можно без всяких вычислений получить описание систем из двух законов сохранения класса Темпля, приведенное в $\S 3$.

В $\S 6$ устанавливается инвариантность систем класса Темпля относительно корреляций трехмерного проективного пространства. 


\section{$\S 2$. Системы законов сохранения. Кривые разрежения и ударная адиабата. Теорема Темпля. Примеры}

Мы рассматриваем системы $n$ законов сохранения

$$
u_{t}^{i}=f^{i}(u)_{x}, \quad i=1, \ldots, n,
$$

предполагая пространственную переменную $x$ одномерной. Начнем со стандартных определений.

Кривые разрежения. Пусть $\lambda^{i}(u), i=1, \ldots, n,-$ собственные числа матрицы $v_{j}^{i}(u)=\frac{\partial f^{i}(u)}{\partial u^{j}}$, называемые характеристическими скоростями системы (1), $\vec{\xi}_{i}$ - отвечающие им собственные векторы:

$$
v \vec{\xi}_{i}=\lambda^{i} \vec{\xi}_{i}
$$

Мы предполагаем, что в рассматриваемой области переменных $u^{i}$ система (1) является гиперболической, т. е. все ее характеристические скорости $\lambda^{i}$ вещественны и различны. Интегральные кривые векторного поля $\vec{\xi}_{i}$ носят название кривы $x$ разрежения $i$-го семейства. Через каждую точку $u_{0}$ пространства зависимых переменных проходит ровно $n$ кривых разрежения - по одной кривой из каждого семейства.

Ударная адиабата. Для фиксированной точки $u_{0}=\left(u_{0}^{1}, \ldots, u_{0}^{n}\right)$ рассмотрим множество всех таких $u=\left(u^{1}, \ldots, u^{n}\right)$, что

$$
\sigma\left(u^{i}-u_{0}^{i}\right)+f^{i}(u)-f^{i}\left(u_{0}\right)=0, \quad i=1, \ldots, n,
$$

для некоторой функции $\sigma\left(u, u_{0}\right)$. Говорят, что $(2)$ является уравнением ударной адиабаты с вершиной в точке $u_{0}$. Для всякого $u$ на ударной адиабате разрывная функция

$$
\begin{array}{ll}
u(x, t)=u_{0}, & x<\sigma t, \\
u(x, t)=u, & x>\sigma t,
\end{array}
$$

является слабым решением системы законов сохранения (1). Как было показано П.О. Лаксом [2], ударная адиабата с вершиной в точке $u_{0}$ общего положения распадается на $n$ ветвей, каждая из которых имеет $C^{2}$-касание с соответствуюшей кривой разрежения $i$-го семейства, проходяшей через точку $u_{0}$.

Кривые разрежения и ударная адиабата играют фундаментальную роль в теории слабых решений систем законов сохранения; их геометрия полностью определяет сценарий распада произвольного начального разрыва.

Как отмечалось в ряде работ, возможно тождественное совпадение ударной адиабаты и соответствующей кривой разрежения. Именно такая ситуация возникает, например, в теории многокомпонентной хроматографии с ленгмюровской изотермой сорбции. Явление совпадения ударной адиабаты и кривых разрежения подробно изучалось Темплем в [3]. Основной результат формулируется в виде следующей теоремы. 
Теорема 1 [3]. Кривые разрежения $i$-го семейства совпадают с соответствующими им ветвями ударной адиабаты в том и только том случае, если имеет место альтернатива:

1) все кривые разрежения $i$-го семейства являются прямыми линиями в и-пространстве;

2) характеристическая скорость $\lambda^{i}$ постоянна вдоль кривых разрежения $i$-го семейства:

$$
L_{i}\left(\lambda^{i}\right)=0,
$$

где $L_{i}=L_{\vec{\xi}_{i}}-$ производная Ли вдоль собственного вектора $\vec{\xi}_{i}$.

В теории квазилинейных систем условие 2 ) известно как условие слабой нелинейности [1].

ОПРеДЕлЕниЕ 1. Системы законов сохранения (1), у которых для любого $i=1, \ldots, n$ имеет место альтернатива 1$), 2)$, мы будем называть системами класса Темпля.

ПримеР 1. Рассмотрим систему уравнений хроматографиии [1]:

$$
u_{t}^{i}+\left(\frac{\Gamma_{i} u^{i}}{V}\right)_{x}=0, \quad i=1, \ldots, n
$$

где $\Gamma_{i}$ - const, $V=1+\sum_{k=1}^{n} u^{k}$. Характеристические скорости $\lambda^{i}$ являются корнями уравнения $n$-й степени

$$
\frac{\Gamma_{1} u^{1}}{\Gamma_{1}-\lambda V}+\cdots+\frac{\Gamma_{n} u^{n}}{\Gamma_{n}-\lambda V}=V
$$

В простейшем случае $n=2$ имеем

$$
\frac{\Gamma_{1} u^{1}}{\Gamma_{1}-\lambda V}+\frac{\Gamma_{2} u^{2}}{\Gamma_{2}-\lambda V}=V, \quad V=1+u^{1}+u^{2} .
$$

Условие равенства нулю дискриминанта этого квадратного (по $\lambda$ ) уравнения имеет вид

$$
\left[\Gamma_{1}\left(u^{2}+1\right)+\Gamma_{2}\left(u^{1}+1\right)\right]^{2}=4 \Gamma_{1} \Gamma_{2}\left(1+u^{1}+u^{2}\right)
$$

и задает параболу на плоскости зависимых переменных $u^{1}, u^{2}$. Через каждую точку плоскости, лежащую вне этой параболы, проходят ровно две касательные к параболе. Несложно показать, что они и являются кривыми разрежения нашей системы, в то время как внешность параболы является ее областью гиперболичности.

В случае $n=3$ характеристическое уравнение имеет вид

$$
\frac{\Gamma_{1} u^{1}}{\Gamma_{1}-\lambda V}+\frac{\Gamma_{2} u^{2}}{\Gamma_{2}-\lambda V}+\frac{\Gamma_{3} u^{3}}{\Gamma_{3}-\lambda V}=V, \quad V=1+u^{1}+u^{2}+u^{3} .
$$

Несложный анализ показывает, что условие равенства нулю дискриминанта этого кубического по $\lambda$ уравнения задает двумерную развертывающуюся поверхность в 
пространстве зависимых переменных $u^{1}, u^{2}, u^{3}$, ребром возврата которой служит кривая

$$
\begin{aligned}
& u^{1}=\left(\Gamma_{1} s-1\right)^{3} \frac{\Gamma_{2} \Gamma_{3}}{\left(\Gamma_{1}-\Gamma_{2}\right)\left(\Gamma_{1}-\Gamma_{3}\right)}, \\
& u^{2}=\left(\Gamma_{2} s-1\right)^{3} \frac{\Gamma_{1} \Gamma_{3}}{\left(\Gamma_{2}-\Gamma_{1}\right)\left(\Gamma_{2}-\Gamma_{3}\right)}, \\
& u^{3}=\left(\Gamma_{3} s-1\right)^{3} \frac{\Gamma_{1} \Gamma_{2}}{\left(\Gamma_{3}-\Gamma_{1}\right)\left(\Gamma_{3}-\Gamma_{2}\right)},
\end{aligned}
$$

параметризованная параметром $s$. На ребре возврата совпадают все три характеристические скорости, в то время как в других точках дискриминанта совпадает лишш какая-либо пара из них. Через каждую точку в $u$-пространстве проходят ровно три плоскости, касающиеся дискриминанта (напомним, что касательная плоскость к развертывающейся поверхности касается ее по всей прямолинейной образуюшей и является соприкасающейся плоскостью ребра возврата).

Эти три плоскости есть не что иное как поверхности уровня соответствующих инвариантов Римана. Три прямые линии попарных пересечений этих плоскостей и являются кривыми разрежения нашей системы. Как легко видеть, каждая линия разрежения касается дискриминанта в двух точках. Геометрия линий разрежения системы уравнений изотахофореза, близкой по своим свойствам к системе (3), подробно изучалась в [4] при исследовании задачи распада произвольного начального разрыва.

Поскольку система уравнений хроматографии имеет прямолинейные кривые разрежения, она принадлежит классу Темпля. При этом ни одна из ее характеристических скоростей $\lambda^{i}$ не является слабо нелинейной.

ПРимеР 2. Система трех уравнений

$$
\begin{gathered}
a_{t}=b_{x}, \\
b_{t}=c_{x}, \\
c_{t}=\left(b^{2}-a c\right)_{x}
\end{gathered}
$$

возникла в теории уравнений ассоциативности двумерной топологической теории поля [5] и подробно изучалась в [6]-[8]. Ее характеристические скорости $\lambda^{i}$ являются корнями кубического уравнения

$$
\lambda^{3}+a \lambda^{2}-2 b \lambda+c=0
$$

Равенство нулю дискриминанта этого уравнения задает двумерную развертывающуюся поверхность в пространстве переменных $a, b, c: 27 q^{2}-4 p^{3}=0$, где $p=2 b+\frac{a^{2}}{3}, q=\frac{2}{27} a^{3}+\frac{2}{3} a b+c$, ребром возврата которой служит норм-кривая

$$
a=3 s, \quad b=-\frac{3}{2} s^{2}, \quad c=s^{3},
$$

параметризованная параметром $s$. Через каждую точку в $(a, b, c)$-пространстве проходят ровно три плоскости, касающиеся дискриминанта. В каждой из этих 
плоскостей проведем прямую, проходящую через точку $(a, b, c)$ параллельно прямолинейной образующей, по которой данная плоскость касается дискриминанта. Полученные в результате такой конструкции три прямые, проходящие через точку $(a, b, c)$, являются линиями разрежения нашей системы.

Подчеркнем, что в данном случае касательные плоскости к дискриминанту не имеют прозрачного смысла поверхностей уровня инвариантов Римана, подобно тому как это имело место в случае уравнений хроматографии (3). Дело в том, что согласно [6]-[8] система (4) является недиагонализуемой. Поскольку система (4) является слабо нелинейной (см. [6]-[8]), она может быть названа “дважды” темплевской - для нее оказываются одновременно выполненными оба пункта альтернативы Темпля.

Отметим, что дискриминанты из примеров 1 и 2 являются аффинно эквивалентными, поскольку аффинно эквивалентны их ребра возврата (оба сводятся к стандартной норм-кривой $\left(s, s^{2}, s^{3}\right)$ подходящим аффиинным преобразованием). Несмотря на это обстоятельство геометрия линий разрежения в обоих примерах существенно различна.

\section{§ 3. Преобразования по решению систем законов} сохранения. Инвариантность систем класса Темпля

Рассмотрим произвольную систему гидродинамического типа

$$
u_{t}^{i}=v_{j}^{i}(u) u_{x}^{j}, \quad i, j=1, \ldots, n,
$$

и пару ее законов сохранения $B(u) d x+A(u) d t$ и $N(u) d x+M(u) d t$, понимаемых как 1-формы, замкнутые в силу уравнений (5), т. е. $B(u)_{t}=A(u)_{x}, N(u)_{t}=$ $M(u)_{x}$. Переходя к новым независимым переменным $X, T$ по формулам

$$
d X=B(u) d x+A(u) d t, \quad d T=N(u) d x+M(u) d t
$$

мы перепишем уравнения (5) в виде

$$
u_{T}^{i}=V_{j}^{i}(u) u_{X}^{j}, \quad i, j=1, \ldots, n
$$

где матрища $V$ связана с $v$ формулой

$$
V=(B v-A E)(M E-N v)^{-1}, \quad E=\mathrm{id} .
$$

Преобразования (6) носят название преобразований по решению [1], в зарубежной литературе - reciprocal transformations [9], [10]. Для систем гидродинамического типа преобразования по решению и их инварианты подробно обсуждались в [11], [12].

Из формулы (8) автоматически вытекает следующая зависимость между характеристическими скоростями $\Lambda^{i}(u)$ системы $(7)$ и характеристическими скоростями $\lambda^{i}(u)$ системы (5):

$$
\Lambda^{i}=\frac{\lambda^{i} B-A}{M-\lambda^{i} N} .
$$


Отметим также, что преобразования по решению не меняют собственных векторов матрицы $v$ и, следовательно, сохраняют кривые разрежения.

Предположим, что исходная система (5) записана в консервативной форме (1):

$$
u_{t}^{i}=f^{i}(u)_{x}, \quad i=1, \ldots, n
$$

т. е. изначально фиксированы $n$ законов сохранения

$$
u^{i} d x+f^{i} d t
$$

Выражая $d x$ и $d t$ через $d X$ и $d T$ из уравнений (6) и подставляя полученные выражения в (9), мы получим преобразованные законы сохранения

$$
U^{i} d X+F^{i} d T
$$

где

$$
U^{i}=\frac{u^{i} M-f^{i} N}{B M-A N}, \quad F^{i}=\frac{f^{i} B-u^{i} A}{B M-A N},
$$

и, соответственно, преобразованную систему (1):

$$
U_{T}^{i}=F^{i}(U)_{X}, \quad i=1, \ldots, n .
$$

ПРЕДЛОЖЕНИЕ 1 [13]. Если Характеристическая скорость $\lambda^{i}$ системы (5) является слабо нелинейной, то такой жее является и соответствующая ей характеристическая скорость $\Lambda^{i}$ системы (7), т. е. преобразования по решению сохраняют свойство слабой нелинейности.

Хотя прямолинейность кривых разрежения, вообще говоря, не сохраняется при произвольных преобразованиях по решению (6), имеется достаточно широкий класс преобразований, которые сохраняют и это свойство, - достаточно взять в качестве интегралов (6) произвольные линейные комбинации исходных интегралов (9):

$$
\begin{aligned}
d X & =B d x+A d t=\left(\alpha_{i} u^{i}+\alpha\right) d x+\left(\alpha_{i} f^{i}+\tilde{\alpha}\right) d t, \\
d T & =N d x+M d t=\left(\beta_{i} u^{i}+\beta\right) d x+\left(\beta_{i} f^{i}+\tilde{\beta}\right) d t,
\end{aligned}
$$

где $\alpha_{i}, \beta_{i}, \alpha, \beta, \tilde{\alpha}, \tilde{\beta}$ - константы. Начиная с этого момента, мы будем рассматривать только преобразования по решению этого специального вида.

Теорема 2. Системы законов сохранения класса Темпля переходят в системы класса Темпля при произвольных преобразованиях (12).

ДокАЗАтЕльство. В силу предложения 1 нам остается показать, что при преобразованиях (12) сохраняется прямолинейность кривых разрежения.

Пусть $\lambda^{i}$-характеристическая скорость, $\vec{\xi}_{i}$ - отвечающий ей собственный вектор системы (1) такие, что соответствующая кривая разрежения является прямой линией. Пусть $u=\left(u^{1}, \ldots, u^{n}\right)$ и $f=\left(f^{1}, \ldots, f^{n}\right)$ - векторы, составленные из плотностей и токов законов сохранения (9). Как известно (см., например, [13]), для плотностей и токов законов сохранения имеет место равенство

$$
L_{i} f=\lambda^{i} L_{i} u
$$


для любого $i=1, \ldots, n$ (напомним, что $L_{i}$ обозначает производную Ли вдоль собственного вектора $\vec{\xi} i$, которая применяется покомпонентно к векторам $f$ и $u$ ).

Условие прямолинейности $i$-й кривой разрежения имеет вид

$$
L_{i}^{2} u=a_{i} L_{i} u
$$

для некоторого скаляра $a_{i}(u)$. В самом деле, $L_{i} u$ есть не что иное как касательньй вектор к $i$-й кривой разрежения, а условие коллинеарности $L_{i}^{2} u$ и $L_{i} u$ есть условие ее прямолинейности.

Нам требуется показать, что

$$
L_{i}^{2} U=A_{i} L_{i} U
$$

(здесь $U$ вычисляется по формуле $(10))$ с некоторым новым коэффициентом пропорциональности $A_{i}$. В целях сокращения вычислений мы установим справедливость (15) для двух частных типов преобразований (12).

I тип (меняется только независимая переменная $x$ ):

$$
d X=B d x+A d t, \quad d T=d t .
$$

В этом случае (10) приводит к $U=\frac{1}{B} u$ и

$$
\begin{aligned}
L_{i} U & =\frac{1}{B} L_{i} u-\frac{L_{i} B}{B^{2}} u, \\
L_{i}^{2} U & =\frac{1}{B} L_{i}^{2} u-2 \frac{L_{i} B}{B^{2}} L_{i} u-\frac{L_{i}^{2} B}{B^{2}} u+2 \frac{\left(L_{i} B\right)^{2}}{B^{3}} u .
\end{aligned}
$$

Поскольку

$$
L_{i}^{2} u=a_{i} L_{i} u, \quad L_{i}^{2} B=a_{i} L_{i} B
$$

имеем

$$
L_{i}^{2} U=\left(a_{i}-2 \frac{L_{i} B}{B}\right) L_{i} U
$$

т. е. векторы $L_{i}^{2} U$ и $L_{i} U$ коллинеарны.

II тип (замена $x$ на $t$ ):

$$
d X=d t, \quad d T=d x
$$

В этом случае $U=f$ и

$$
\begin{aligned}
& L_{i} U=L_{i} f=\lambda^{i} L_{i} u \\
& L_{i}^{2} U=\lambda^{i} L_{i}^{2} u+L_{i} \lambda^{i} L_{i} u=\left(a_{i}+\frac{L_{i} \lambda^{i}}{\lambda^{i}}\right) L_{i} U
\end{aligned}
$$

что вновь доказывает коллинеарность векторов $L_{i}^{2} U$ и $L_{i} U$.

Поскольку комбинациями преобразований типов I и II можно получить любое преобразование вида (12), теорема 2 доказана.

Фундаментальным свойством преобразований (12) является свойство инвариантности ударной адиабаты. 
Теорема 3. Ударная адиабата системы (1)

$$
\sigma\left(u^{i}-u_{0}^{i}\right)+f^{i}(u)-f^{i}\left(u_{0}\right)=0
$$

переходит в ударную адиабату системы (11)

$$
\tilde{\sigma}\left(U^{i}-U_{0}^{i}\right)+F^{i}(U)-F^{i}\left(U_{0}\right)=0
$$

при произвольных преобразованиях по решению (12).

ДокАЗАТЕЛЬСтво. Как и при доказательстве теоремы (2), мы продемонстрируем инвариантность ударной адиабаты для двух частных типов преобразований (12).

I тип (меняется только независимая переменная $x$ ):

$$
d X=B d x+A d t, \quad d T=d t .
$$

В этом случае из (10) следует, что $U^{i}=\frac{1}{B} u^{i}, F^{i}=f^{i}-\frac{A}{B} u^{i}$. Требуется показать, что уравнения

$$
\tilde{\sigma}\left(U^{i}-U_{0}^{i}\right)+F^{i}(U)-F^{i}\left(U_{0}\right)=0
$$

или же

$$
\tilde{\sigma}\left(\frac{u^{i}}{B}-\frac{u_{0}^{i}}{B_{0}}\right)+f^{i}-\frac{A}{B} u^{i}-\left(f_{0}^{i}-\frac{A_{0}}{B_{0}} u_{0}^{i}\right)=0
$$

являются следствиями уравнений

$$
\sigma\left(u^{i}-u_{0}^{i}\right)+f^{i}(u)-f^{i}\left(u_{0}\right)=0
$$

при подходящем выборе коэффициента $\tilde{\sigma}$. Заметим, что, поскольку закон сохранения $B d x+A d t$ является линейной комбинацией законов сохранения (9), имеем также

$$
\sigma\left(B-B_{0}\right)+A-A_{0}=0 .
$$

Подставляя в (17) выражения для $f^{i}$ и $A$, полученные из (18) и (19), мы после элементарных преобразований приходим к равенству

$$
\left(\tilde{\sigma}-\sigma B_{0}-A_{0}\right)\left(\frac{u^{i}}{B}-\frac{u_{0}^{i}}{B_{0}}\right)=0
$$

откуда $\tilde{\sigma}=\sigma B_{0}+A_{0}$. Итак, уравнения (16) являются следствием (18) при выборе $\tilde{\sigma}=\sigma B_{0}+A_{0}$, что и требовалось доказать.

II тип (замена $x$ на $t$ ):

$$
d X=d t, \quad d T=d x .
$$

В этом случае $U^{i}=f^{i}, F^{i}=u^{i}$ и уравнения ударной адиабаты в переменных $U^{i}$

$$
\tilde{\sigma}\left(U^{i}-U_{0}^{i}\right)+F^{i}(U)-F^{i}\left(U_{0}\right)=0
$$

очевидным образом эквивалентны уравнениям ударной адиабаты в переменных $u^{i}$

$$
\sigma\left(u^{i}-u_{0}^{i}\right)+f^{i}(u)-f^{i}\left(u_{0}\right)=0
$$

при выборе $\tilde{\sigma}=1 / \sigma$. 
Поскольку комбинации преобразований типов I и II порождают произвольное преобразование по решению (12), теорема 3 доказана.

Теорема 2 является теперь элементарным следствием теоремы 3 . Действительно, поскольку как кривые разрежения, так и ударная адиабата инвариантны относительно преобразований (12), то и класс систем Темпля, характеризующийся их совпадением, также является инвариантным. Такой способ доказательства теоремы 2 представляется интересным и сам по себе, поскольку фактически не аппелирует к теореме 1 .

ЗАмЕчАниЕ. Вообще говоря, ударная адиабата определяется выбором консервативной записи, и если взять интегралы, не являющиеся линейными комбинациями (9) (а у системы могут быть такие), то изменится и уравнение адиабаты. Другим проявлением этого факта является то, что адиабата не инвариантна относительно произвольных преобразований по решению (6). Объяснение заключается в том, что для слабых решений, определенных консервативной записью (1), произвольное преобразование по решению (6), вообше говоря, не определено, т.е. интеграл от форм

$$
B(u) d x+A(u) d t, \quad N(u) d x+M(u) d t
$$

будет зависеть от пути интегрирования.

Простейшим проявлением теоремы 3 является хорошо известный в газовой динамике факт инвариантности ударной адиабаты при переходе от эйлеровых координат к массовым лагранжевым. Заметим, что переход к лагранжевым координатам есть пример преобразования типа (12).

Свойства инвариантности кривых разрежения, ударной адиабаты и систем класса Темпля указывают на то, что преобразования по решению (12) играют важную роль в теории систем законов сохранения. Как мы увидим в дальнейшем, наряду с аффинными преобразованиями переменных $u^{i}$ они порождают конечномерную группу Ли, изоморфную проективной групте $\mathrm{SL}(n+2, R)$.

\section{§4. Системы из двух законов сохранения класса Темпля}

Согласно теореме 1 системы из двух законов сохранения класса Темпля

$$
\begin{aligned}
& u_{t}^{1}=f^{1}(u)_{x}, \\
& u_{t}^{2}=f^{2}(u)_{x}
\end{aligned}
$$

естественным образом разбиваются на три типа.

I тип. Кривые разрежения обоих семейств являются прямыми.

II тип. Характеристические скорости обоих семейств являются слабо нелинейньми.

III тип. Кривые разрежения одного семейства являются прямыми, а характеристические скорости другого семейства являются слабо нелинейными.

В настоящем параграфе приводится явный вид правых частей $f^{1}, f^{2}$ для систем каждого из трех типов. Перед тем как сформулировать ответ, поясним, как могут 
быть получены уравнения на $f^{1}, f^{2}$, характеризующие, например, системы типов I и II. Для этого рассмотрим характеристическое уравнение системы (20)

$$
\lambda^{2}-\left(f_{1}^{1}+f_{2}^{2}\right) \lambda+\left(f_{1}^{1} f_{2}^{2}-f_{2}^{1} f_{1}^{2}\right)=0
$$

где введены обозначения $f_{j}^{i}=\frac{\partial f^{i}}{\partial u^{j}}$. Собственный вектор $\vec{\xi}$, отвечающий характеристической скорости $\lambda$, выберем в виде

$$
\vec{\xi}=f_{2}^{1} \frac{\partial}{\partial u^{1}}+\left(\lambda-f_{1}^{1}\right) \frac{\partial}{\partial u^{2}} .
$$

Системы типов I и II выделяются условиями

$$
L_{\vec{\xi}}\left(\frac{\lambda-f_{1}^{1}}{f_{2}^{1}}\right)=0
$$

и

$$
L_{\vec{\xi}}(\lambda)=0
$$

соответственно, где $L \vec{\xi}-$ производная Ли вдоль векторного поля (22). Заметим, что поскольку $\frac{\partial \lambda}{\partial u^{1}}$ и $\frac{\partial \lambda}{\partial u^{2}}$ находятся дифференцированием характеристического уравнения (21), оба выражения (23) и (24) могут быть приведены к полиномам первой степени по $\lambda$ (так как $\lambda^{2}, \lambda^{3}$ и т. д. выражаются через $\lambda$ в силу характеристического уравнения (21)), коэффициенты которых зависят от $f^{1}, f^{2}$ и их производных до второго порядка включительно. Приравнивание к нулю коэффициентов этих полиномов и дает искомые уравнения на $f^{1}, f^{2}$.

I тип. Соотношение (23) приводит к следующей системе двух уравнений второго порядка на функции $f^{1}, f^{2}$ :

$$
\begin{aligned}
& f_{2}^{1} f_{1}^{2}\left(2 f_{12}^{2}-f_{11}^{1}\right)+f_{2}^{1}\left(f_{1}^{1}-f_{2}^{2}\right) f_{11}^{2}-\left(f_{1}^{2}\right)^{2} f_{22}^{1}=0 \\
& f_{1}^{2} f_{2}^{1}\left(2 f_{12}^{1}-f_{22}^{2}\right)+f_{1}^{2}\left(f_{2}^{2}-f_{1}^{1}\right) f_{22}^{1}-\left(f_{2}^{1}\right)^{2} f_{11}^{2}=0
\end{aligned}
$$

(нижние индексы обозначают дифференцирование по переменным $u^{1}, u^{2}$ ). Обший интеграл этой системы, найденный в [3], дается неявными формулами

$$
\begin{array}{ll}
u^{1}=\frac{g^{1}(p)-g^{2}(q)}{q-p}, & u^{2}=\frac{q g^{1}(p)-p g^{2}(q)}{q-p}, \\
f^{1}=\frac{h^{1}(p)-h^{2}(q)}{q-p}, & f^{2}=\frac{q h^{1}(p)-p h^{2}(q)}{q-p},
\end{array}
$$

где $g^{1}(p), g^{2}(q), h^{1}(p), h^{2}(q)$ - четыре произвольные функции своих аргументов. Выражая $p, q$ как функции $u^{1}, u^{2}$ и подставляя их в формулы для $f^{1}, f^{2}$, мы получим общее решение системы (25). Заметим, что $p, q$ можно эквивалентным образом задать как решения уравнений

$$
p_{1}+p p_{2}=0, \quad q_{1}+q q_{2}=0 .
$$


II тип. Соотношения (24) приводят к следуюшей системе двух уравнений второго порядка на функции $f^{1}, f^{2}$ :

$$
\begin{aligned}
& \left(f_{2}^{2}-f_{1}^{1}\right) f_{11}^{1}-2 f_{1}^{2} f_{12}^{1}-f_{1}^{2} f_{22}^{2}-f_{2}^{1} f_{11}^{2}=0 \\
& \left(f_{1}^{1}-f_{2}^{2}\right) f_{22}^{2}-2 f_{2}^{1} f_{12}^{2}-f_{2}^{1} f_{11}^{1}-f_{1}^{2} f_{22}^{1}=0
\end{aligned}
$$

общий интеграл которой дается неявными формулами

$$
\begin{array}{rlrl}
u^{1} & =\frac{g^{1}(p)-g^{2}(q)}{q-p}, & u^{2} & =\frac{h^{1}(p)-h^{2}(q)}{q-p}, \\
f^{1} & =\frac{q g^{1}(p)-p g^{2}(q)}{q-p}, & f^{2}=\frac{q h^{1}(p)-p h^{2}(q)}{q-p}
\end{array}
$$

где $g^{1}(p), g^{2}(q), h^{1}(p), h^{2}(q)$ - четыре произвольные функции своих аргументов.

Сравнивая формулы (26) и (28), мы видим, что точечная замена

$$
u^{1} \rightarrow u^{1}, \quad u^{2} \rightarrow f^{1}, \quad f^{1} \rightarrow u^{2}, \quad f^{2} \rightarrow f^{2}
$$

переводит системы (25) и (27) друг в друга.

Проективно-геометрическая интерпретация этого странного на первый взгляд факта будет дана в $\S 6$.

III тип. Для систем этого типа уравнения на $f^{1}, f^{2}$ имеют более сложный вид

$$
\begin{gathered}
2 f_{12}^{1}\left(f_{1}^{2} f_{22}^{2}+f_{2}^{1} f_{11}^{2}\right)-2 f_{12}^{2}\left(f_{2}^{1} f_{11}^{1}+f_{1}^{2} f_{22}^{1}\right)=\left(f_{2}^{2}-f_{1}^{1}\right)\left(f_{11}^{1} f_{22}^{2}-f_{22}^{1} f_{11}^{2}\right) \\
f_{2}^{1}\left(f_{1}^{2} f_{22}^{2}+f_{2}^{1} f_{11}^{2}\right)^{2}-f_{1}^{2}\left(f_{2}^{1} f_{11}^{1}+f_{1}^{2} f_{22}^{1}\right)^{2} \\
=\left(f_{2}^{2}-f_{1}^{1}\right)\left(f_{1}^{2} f_{22}^{2}+f_{2}^{1} f_{11}^{2}\right)\left(f_{2}^{1} f_{11}^{1}+f_{1}^{2} f_{22}^{1}\right)
\end{gathered}
$$

обшее решение которых (найденное в [3] без выписьвания самих формул (30)) имеет вид (с точностью до сдвигов $u^{i} \rightarrow u^{i}+$ const, $f^{i} \rightarrow f^{i}+$ const)

$$
f^{1}=u^{1} g\left(u^{1}, u^{2}\right), \quad f^{2}=u^{2} g\left(u^{1}, u^{2}\right)
$$

где $g\left(u^{1}, u^{2}\right)$ - произвольная функция. Любопытно, что системы Темпля типа III зависят от одной функции двух аргументов, в то время как системы типов I и II лишш от четырех функций одного аргумента. В $\S 5$ будет показано, как получить формулы $(26),(28)$ и (31) на основе простых геометрических соображений. 
Tеорема 4. Уравнения (25), (27) и (30) допускают одну и ту же 15-параметрическую группу Ли точечных симметрий с инфинитезимальными операторами

$$
\begin{gathered}
T U_{1}=\frac{\partial}{\partial u^{1}}, \quad T U_{2}=\frac{\partial}{\partial u^{2}}, \quad T F_{1}=\frac{\partial}{\partial f^{1}}, \quad T F_{2}=\frac{\partial}{\partial f^{2}} \\
D_{0}=u^{1} \frac{\partial}{\partial u^{1}}+u^{2} \frac{\partial}{\partial u^{2}}, \quad D_{1}=f^{1} \frac{\partial}{\partial f^{1}}+f^{2} \frac{\partial}{\partial f^{2}}, \quad D_{2}=u^{2} \frac{\partial}{\partial u^{2}}+f^{2} \frac{\partial}{\partial f^{2}} \\
R_{1}=f^{1} \frac{\partial}{\partial u^{1}}+f^{2} \frac{\partial}{\partial u^{2}}, \quad R_{2}=u^{1} \frac{\partial}{\partial f^{1}}+u^{2} \frac{\partial}{\partial f^{2}} \\
R_{1,2}=u^{2} \frac{\partial}{\partial u^{1}}+f^{2} \frac{\partial}{\partial f^{1}}, \quad R_{2,1}=u^{1} \frac{\partial}{\partial u^{2}}+f^{1} \frac{\partial}{\partial f^{2}} \\
P U_{1}=\left(u^{1}\right)^{2} \frac{\partial}{\partial u^{1}}+u^{1} u^{2} \frac{\partial}{\partial u^{2}}+u^{1} f^{1} \frac{\partial}{\partial f^{1}}+u^{2} f^{1} \frac{\partial}{\partial f^{2}} \\
P U_{2}=u^{1} u^{2} \frac{\partial}{\partial u^{1}}+\left(u^{2}\right)^{2} \frac{\partial}{\partial u^{2}}+u^{1} f^{2} \frac{\partial}{\partial f^{1}}+u^{2} f^{2} \frac{\partial}{\partial f^{2}} \\
P F_{1}=u^{1} f^{1} \frac{\partial}{\partial u^{1}}+u^{1} f^{2} \frac{\partial}{\partial u^{2}}+\left(f^{1}\right)^{2} \frac{\partial}{\partial f^{1}}+f^{1} f^{2} \frac{\partial}{\partial f^{2}} \\
P F_{2}=u^{2} f^{1} \frac{\partial}{\partial u^{1}}+u^{2} f^{2} \frac{\partial}{\partial u^{2}}+f^{1} f^{2} \frac{\partial}{\partial f^{1}}+\left(f^{2}\right)^{2} \frac{\partial}{\partial f^{2}}
\end{gathered}
$$

Операторы (32) образуют, как мы увидим в $\$ 4$, алгебру Ли, изоморфную $\mathrm{SL}(4, R)$.

Инвариантность уравнений (25), (27) и (30) относительно операторов (32) есть не что иное как отражение инвариантности систем класса Темпля относительно преобразований по решению (12), аффинных замен $u^{i}$ и сдвигов $f^{i}$ (см. теорему 2). Как видно из таблицы, приведенной в приложении, все конечные преобразования, отвечающие инфинитезимальным операторам (32), порождаются либо подходящими преобразованиями по решению (12) системы (20), либо аффинными преобразованиями переменных $u^{i}, f^{i}$.

Все конструкции настоящего параграфа в принципе могут быть перенесены на системы $n$ законов сохранения класса Темпля для любого $n>2$. Однако уже для $n=3$ аналоги уравнений $(25),(27)$ и (30) становятся чрезвычайно громоздкими. Тем не менее все эти уравнения будут допускать $\left((n+2)^{2}-1\right)$-параметрическую группу Ли точечных симметрий с инфинитезимальными операторами

$$
\begin{aligned}
T U_{j} & =\frac{\partial}{\partial u^{j}}, \quad T F_{j}=\frac{\partial}{\partial f^{j}}, \quad j=1, \ldots, n, \\
D_{0}=\sum_{i=1}^{n} u^{i} \frac{\partial}{\partial u^{i}}, \quad D_{1} & =\sum_{i=1}^{n} f^{i} \frac{\partial}{\partial f^{i}}, \quad D_{j}=u^{j} \frac{\partial}{\partial u^{j}}+f^{j} \frac{\partial}{\partial f^{j}}, \quad j=2, \ldots, n, \\
R_{1} & =\sum_{i=1}^{n} f^{i} \frac{\partial}{\partial u^{i}}, \quad R_{2}=\sum_{i=1}^{n} u^{i} \frac{\partial}{\partial f^{i}},
\end{aligned}
$$




$$
\begin{aligned}
& R_{j, k}=u^{k} \frac{\partial}{\partial u^{j}}+f^{k} \frac{\partial}{\partial f^{j}}, \quad j, k=1, \ldots, n, \quad j \neq k, \\
& P U_{j}=u^{j} \sum_{i=1}^{n} u^{i} \frac{\partial}{\partial u^{i}}+f^{j} \sum_{i=1}^{n} u^{i} \frac{\partial}{\partial f^{i}}, \quad j=1, \ldots, n, \\
& P F_{j}=u^{j} \sum_{i=1}^{n} f^{i} \frac{\partial}{\partial u^{i}}+f^{j} \sum_{i=1}^{n} f^{i} \frac{\partial}{\partial f^{i}}, \quad j=1, \ldots, n,
\end{aligned}
$$

которые очевидным образом обобщают операторы (32). Наличие этой алгебры Ли, изоморфной, как мы покажем, $\mathrm{SL}(n+2, R)$, является следствием инвариантности систем класса Темпля относительно преобразований по решению (12), аф̆финных замен $u^{i}$ и сдвигов $f^{i}$.

\section{$\S 5$. Соответствие между системами законов сохранения и семействами прямых. Основные свойства}

Всякой системе $n$ законов сохранения (1)

$$
u_{t}^{i}=f^{i}(u)_{x}, \quad i=1, \ldots, n,
$$

мы сопоставим $n$-параметрическое семейство прямых в $(n+1)$-мерном пространстве $A^{n+1}$ с координатами $y^{0}, y^{1}, \ldots, y^{n}$, заданное уравнениями

$$
\begin{gathered}
y^{1}=u^{1} y^{0}-f^{1}(u), \\
\ldots \ldots \ldots \ldots \ldots \\
y^{n}=u^{n} y^{0}-f^{n}(u)
\end{gathered}
$$

(переменные $u^{1}, \ldots, u^{n}$ интерпретируются как параметры семейства (34)). Заметим, что всякое $n$-параметрическое семейство прямых обшего положения может быть записано в виде (34). При $n=2$ мы имеем двухпараметрическое семейство, или, как говорят, конгруэнцию прямых в $A^{n+1}$. Начиная с работ Монжа, Плюккера и Куммера, теория конгруэнций была популярным предметом исследований в классической дифференциальной геометрии. Изложение основ этой теории и подробная библиографиия могут быть найдены в [14].

Оказывается, что многие понятия и конструкции теории систем законов сохранения имеют прозрачную интерпретацию на языке теории конгруэнций.

ТЕорема 5. Преобразованиям по решению (12) и аффинныцм заменам и системы законов сохранения отвечают проективные преобразования семейства прямых (34).

ДокАЗАТЕЛЬСТво. Как отмечалось в $\S 4$, в случае $n=2$ преобразования по решению (12) и аффинные замены переменных $u^{i}$ находятся во взаимно однозначном соответствии с конечными преобразованиями группы Ли, порожденной инфинитезимальными операторами (32). С другой стороны, как следует из таблицы в приложении, всякое такое конечное преобразование порождается подходяшим проективным преобразованием координат $y^{0}, y^{1}, \ldots, y^{n}$ (и наоборот), что без труда прослеживается для каждого из операторов (32). Хотя все формулы в таблице относятся к случаю $n=2$, их обобшение на произвольные $n$ проводится непосредственно. Теорема доказана. 
СлЕДСТВИЕ. Алгебра Ли с инфинитезимальными операторами (33) изоморфна $\mathrm{SL}(n+2, R)$.

В самом деле, группа проективных преобразований $(n+1)$-мерного пространства изоморфна $\mathrm{SL}(n+2, R)$.

Таким образом, изучение систем законов сохранения с точностью до преобразований по решению (12) есть не что иное как проективная геометрия семейств прямых (34), т. е. изучение свойств $n$-параметрических семейств прямых в $A^{n+1}$, инвариантных относительно проективных преобразований.

ТЕОРема 6. Кривым разрежсения системы законов сохранения (1) отвечают развертывающиеся поверхности семейства прямых (34).

ДокАЗАТЕЛЬСТво. Приведем, следуя [14], стандартное построение развертываюшихся поверхностей семейства прямых (34). Рассмотрим произвольную точку $u=\left(u^{i}\right)$ в пространстве параметров и близкую к ней точку $u+\vec{\xi} \epsilon=\left(u^{i}+\xi^{i} \epsilon\right)$, полученную из $u$ сдвигом в направлении вектора $\vec{\xi}$ на расстояние $\epsilon(\epsilon$ предполагается малым параметром). Уравнения прямых, отвечающих точкам $u$ и $u+\vec{\xi} \epsilon$, имеют вид (34) и

$$
\begin{gathered}
y^{1}=\left(u^{1}+\xi^{1} \epsilon\right) y^{0}-f^{1}(u+\vec{\xi} \epsilon), \\
\ldots \ldots \ldots \ldots \ldots \ldots \ldots \cdots \cdots \cdots \cdots \cdots \cdots \cdots \cdots \cdots \\
y^{n}=\left(u^{n}+\xi^{n} \epsilon\right) y^{0}-f^{n}(u+\vec{\xi} \epsilon)
\end{gathered}
$$

соответственно. $\mathrm{C}$ точностью до бесконечно малых второго порядка по $\epsilon$ уравнения (35) переписываются в виде

$$
\begin{gathered}
y^{1}=u^{1} y^{0}-f^{1}(u)+\epsilon\left(\xi^{1} y^{0}-f_{1}^{1} \xi^{1}-\ldots-f_{n}^{1} \xi^{n}\right)+O\left(\epsilon^{2}\right), \\
\ldots \ldots \ldots \ldots \ldots \ldots \ldots \ldots \ldots \ldots \ldots \ldots \ldots \ldots \ldots \ldots \ldots \ldots \ldots \ldots \ldots \ldots \ldots \ldots \ldots \ldots \\
y^{n}=u^{n} y^{0}-f^{n}(u)+\epsilon\left(\xi^{n} y^{0}-f_{1}^{n} \xi^{1}-\ldots .\right.
\end{gathered}
$$

Условие того, что прямые (34) и (35) пересекаются с точностью до бесконечно малых второго порядка по $\epsilon$, имеет вид

$$
\left(\begin{array}{ccc}
f_{1}^{1} & \ldots & f_{n}^{1} \\
\ldots \ldots & \ldots & \ldots \\
f_{1}^{n} & \ldots & f_{n}^{n}
\end{array}\right)\left(\begin{array}{c}
\xi^{1} \\
\ldots \\
\xi^{n}
\end{array}\right)=y^{0}\left(\begin{array}{c}
\xi^{1} \\
\ldots \\
\xi^{n}
\end{array}\right),
$$

т. е. вектор $\vec{\xi}$ должен быть собственным вектором матрицы Якоби $f_{j}^{i}$ с собственным значением $y^{0}$, равным соответствующей характеристической скорости $\lambda$. Таким образом, при изменении параметров $u$ вдоль кривой разрежения нашей системы, отвечающей характеристической скорости $\lambda$ и являющейся интегральной кривой векторного поля $\vec{\xi}$, мы получим однопараметрическое семейство прямых, которое образует развертьвающуюся поверхность. Ребром возврата этой поверхности служит кривая

$$
\begin{aligned}
& y^{0}=\lambda, \\
& y^{1}=u^{1} \lambda-f^{1}(u), \\
& \cdots \cdots \cdots \cdots \cdots \cdots \cdots \cdots \cdots \\
& y^{n}=u^{n} \lambda-f^{n}(u)
\end{aligned}
$$


(здесь предполагается, что параметры $u$ меняются вдоль кривой разрежения, отвечающей характеристической скорости $\lambda)$.

Итак, всякой кривой разрежения системы (1) отвечает развертывающаяся поверхность системы прямых (34). Поскольку через каждую точку $u$ в пространстве параметров проходит ровно $n$ кривых разрежения, то через каждую прямую семейства (34) проходит ровно $n$ развертывающихся поверхностей, по одной из каждого семейства. Теорема доказана.

Как известно из теории конгруэнций [14], множество ребер возврата развертывающихся поверхностей каждого семейства образует так называемую фокальную гиперповерхность. В нашем случае ее уравнение имеет вид

$$
\begin{aligned}
& y^{0}=\lambda, \\
& y^{1}=u^{1} \lambda-f^{1}(u), \\
& \ldots \ldots \ldots \cdots \cdots \cdots \cdots \\
& y^{n}=u^{n} \lambda-f^{n}(u),
\end{aligned}
$$

причем здесь, в отличие от (37), параметр $u$ пробегает все возможные значения. Поскольку система законов сохранения (1) является гиперболической и имеет $n$ вешественных характеристических скоростей $\lambda$, то соответствуюшее ей семейство прямых (34) также является гиперболическим, т. е. имеет $n$ фокальньх гиперповерхностей (38). Все прямые семейства (34) касаются каждой из фокальных гиперповерхностей. Развертывающиеся поверхности семейства (34) касаются фокальных гиперповерхностей вдоль кривых, образующих фокальную сеть.

ТЕОРемА 7. Ударная адиабата с верииной в точке $u_{0}$ отвечает прямым семейства (34), которые пересекают прямую с параметром $u_{0}$.

ДокАЗАТЕльство. В самом деле, условие пересечения прямых

$$
\begin{aligned}
& y^{1}=u_{0}^{1} y^{0}-f_{0}^{1}, \\
& \ldots \ldots \ldots \ldots \\
& y^{n}=u_{0}^{n} y^{0}-f_{0}^{n}
\end{aligned}
$$

и

$$
\begin{aligned}
& y^{1}=u^{1} y^{0}-f^{1}, \\
& \cdots \ldots \ldots \ldots \ldots \\
& y^{n}=u^{n} y^{0}-f^{n}
\end{aligned}
$$

имеет вид

$$
y^{0}\left(u^{i}-u_{0}^{i}\right)=f^{i}-f_{0}^{i},
$$

что в точности совпадает с уравнением ударной адиабаты (2) при обозначении $y^{0}=-\sigma$. Теорема доказана.

Как отмечалось в $\S 1$, через каждую точку $u_{0}$ пространства параметров проходят $n$ ветвей ударной адиабаты, которые имеют $C^{2}$-касание с соответствующими 
кривыми разрежения. Следовательно, через каждую фиксированную прямую семейства (34) наряду с $n$ развертывающимися поверхностями проходят $n$ линейчатых поверхностей (уже, вообще говоря, не развертывающихся), все прямолинейные образуюшие которых пересекают исходную прямую. Эти линейчатые поверхности имеют $C^{2}$-касание с соответствующими им развертывающимися поверхностями вдоль исходной прямой. Любопытно, что введенные нами линейчатые поверхности, несмотря на их явно проективно-инвариантное определение, как будто бы не рассматривались в классической теории конгруэнщий.

Проективная инвариантность введенных нами линейчатых поверхностей полностью эквивалентна инвариантности ударной адиабаты относительно преобразований по решению (12) в силу теоремы 3. Обратим внимание читателя на то обстоятельство, что будучи абсолютно очевидным геометрически свойство инвариантности ударной адиабаты далеко не так прозрачно с точки зрения систем законов сохранения.

В заключение дадим геометрическую интерпретацию коммутирующим системам. Пусть система законов сохранения (1) коммутирует с системой

$$
u_{\tau}^{i}=g^{i}(u)_{x}, \quad i=1, \ldots, n,
$$

т. е. $u_{t \tau}^{i}=u_{\tau t}^{i}$.

ТЕОРема 8. Фокальные гиперповерхности семейства прямых (34), отвечающего системе (1), и семейства прямых, отвечающего коммутирующей $c$ ней системе (41), находятся попарно в соответствии Петерсона. Другими словами, в соответствующих точках касательные к фокальной сети на фокальной гиперповерхности одного семейства параллельны касательным $к$ фокальной сети на фокальной гиперповерхности другого семейства.

ДокАЗАТЕЛЬство. Пусть $\lambda^{1}, \ldots, \lambda^{n}$ - характеристические скорости системы $(1), \vec{\xi}_{1}, \ldots, \vec{\xi}_{n}$ - отвечающие им собственные векторы. Поскольку необходимым условием коммутации является коммутирование соответствующих матрищ Якоби $f_{j}^{i}$ и $g_{j}^{i}$, система (41) имеет те же самые собственные векторы $\vec{\xi}_{1}, \ldots, \vec{\xi}_{n}$, т. е. те же самые кривые разрежения. Обозначим через $w^{1}, \ldots, w^{n}$ характеристические скорости системы (41). Радиус-векторы фокальных гиперповерхностей, отвечающих (для определенности) собственным значениям $\lambda^{1}$ и $w^{1}$, имеют вид (см. формулы $(38))$

и

$$
\vec{r}_{1}=\left(\begin{array}{c}
\lambda^{1} \\
u^{1} \lambda^{1}-f^{1} \\
\ldots \ldots \ldots \\
u^{n} \lambda^{1}-f^{n}
\end{array}\right)
$$

$$
\vec{R}_{1}=\left(\begin{array}{c}
w^{1} \\
u^{1} w^{1}-g^{1} \\
\cdots \cdots \cdots \\
u^{n} w^{1}-g^{n}
\end{array}\right)
$$

соответственно. Покажем, что векторы $L_{1} \vec{r}_{1}, \ldots, L_{n} \vec{r}_{1}$ параллельны векторам $L_{1} \vec{R}_{1}, \ldots, L_{n} \vec{R}_{1}$ (напомним, что $L_{i}$ обозначает дифференщирование Ли вдоль собственного вектора $\vec{\xi} i$. Это и будет являться доказательством теоремы, поскольку векторы $L_{i} \vec{r}_{1}$ (соответственно $L_{i} \vec{R}_{1}$ ) есть касательные к линиям фокальной 
сети на фокальной гиперповерхности с радиус-вектором $\vec{r}_{1}$ (соответственно $\vec{R}_{1}$ ). Непосредственное вычисление дает

$$
L_{i} \vec{r}_{1}=L_{i} \lambda^{1}\left(\begin{array}{c}
1 \\
u^{1} \\
\cdots \\
u^{n}
\end{array}\right)+\left(\lambda^{1}-\lambda^{i}\right)\left(\begin{array}{c}
0 \\
L_{i} u^{1} \\
\cdots \\
L_{i} u^{n}
\end{array}\right)
$$

и

$$
L_{i} \vec{R}_{1}=L_{i} w^{1}\left(\begin{array}{c}
1 \\
u^{1} \\
\ldots \\
u^{n}
\end{array}\right)+\left(w^{1}-w^{i}\right)\left(\begin{array}{c}
0 \\
L_{i} u^{1} \\
\ldots \\
L_{i} u^{n}
\end{array}\right),
$$

где мы воспользовались соотношениями

$$
L_{i} f=\lambda^{i} L_{i} u, \quad L_{i} g=w^{i} L_{i} u
$$

которые имеют место для законов сохранения

$$
u^{i} d x+f^{i} d t, \quad u^{i} d x+g^{i} d \tau
$$

согласно формуле (13).

При значении индекса $i=1$ параллельность $L_{1} \vec{r}_{1}$ и $L_{1} \vec{R}_{1}$ очевидна. При $i \neq 1$ параллельность $L_{i} \vec{r}_{1}$ и $L_{i} \vec{R}_{1}$ вытекает из соотношения

$$
\frac{L_{i} \lambda^{j}}{\lambda^{i}-\lambda^{j}}=\frac{L_{i} w^{j}}{w^{i}-w^{j}} \quad(i \neq j)
$$

выполненного в силу коммутации систем (1) и (41) (см., например, [15]). Теорема доказана.

Следует отметить, что для гамильтоновых систем гидродинамического типа связь коммутируюших систем с преобразованиями Петерсона и Комбескюра отмечалась ранее в [15], [16].

\section{§6. Геометрия систем класса Темпля. Системы из двух законов сохранения класса Темпля с точки зрения теории конгруэнций}

Согласно теореме 1 система законов сохранения принадлежит классу Темпля в том и только том случае, если для любого значения индекса $i=1, \ldots, n$ либо все кривые разрежения $i$-го семейства являются прямыми, либо характеристическая скорость $\lambda^{i}$ является слабо нелинейной. Приведенные ниже теоремы дают наглядную геометрическую переформулировку условий темплевости.

ТЕОРема 9. Кривые разрежсения $i$-го семейства являются прямыми линиями тогда и только тогда, когда соответствующие им развертьвающиеся поверхности являются плоскими, т. е. их ребра возврата-плоские кривые. 
ДокАЗАТЕЛЬСТво. Поскольку интегральные траектории векторного поля $\vec{\xi}_{i}$, соответствующего характеристической скорости $\lambda^{i}$, являются прямыми линиями в $u$-пространстве, имеет место соотношение (14)

$$
L_{i}^{2} u=a_{i} L_{i} u .
$$

Уравнение ребра возврата развертьвающейся поверхности, отвечающей кривой разрежения $i$-го семейства, имеет вид (37)

$$
\vec{y}=\left(\begin{array}{c}
\lambda^{i} \\
u^{1} \lambda^{i}-f^{1} \\
\cdots \cdots \\
u^{n} \lambda^{i}-f^{n}
\end{array}\right)
$$

Вычислим $L_{i} \vec{y}, L_{i}^{2} \vec{y}$ :

$$
L_{i} \vec{y}=L_{i} \lambda^{i}\left(\begin{array}{c}
1 \\
u^{1} \\
\ldots \\
u^{n}
\end{array}\right), \quad L_{i}^{2} \vec{y}=L_{i}^{2} \lambda^{i}\left(\begin{array}{c}
1 \\
u^{1} \\
\ldots \\
u^{n}
\end{array}\right)+L_{i} \lambda^{i}\left(\begin{array}{c}
0 \\
L_{i} u^{1} \\
\ldots \\
L_{i} u^{n}
\end{array}\right) .
$$

Следовательно, соприкасаюшаяся плоскость к ребру возврата порождена векторами

$$
\left(\begin{array}{c}
1 \\
u^{1} \\
\cdots \\
u^{n}
\end{array}\right), \quad\left(\begin{array}{c}
0 \\
L_{i} u^{1} \\
\cdots \\
L_{i} u^{n}
\end{array}\right)
$$

В силу соотношения (14) последуюшие применения оператора $L_{i}$ к этим векторам выражаются через них самих, т. е. соприкасающаяся плоскость к ребру возврата стационарна. Следовательно, ребро возврата - плоская кривая. Теорема доказана.

СлЕДСТВИЕ. Пусть все кривые разрежения системь (1) являются прямыцми линиями. Тогда все развертыввююиеся поверхности семейства прямых (34) являются плоскими, а фокальнье сети на фокальных гиперповерхностях состоят из плоских линий.

ТеОрема 10. Характеристическая скорость $\lambda^{i}$ является слабо нелинейной тогда и только тогда, когда все развертвивающиеся поверхности, соответствующие кривым разрежения $i$-го семейства, являются коническими, т.е. их прямолинейные образующие пересекаются в одной точке.

ДокАЗАТЕЛЬСТво. Вновь рассмотрим ребро возврата развертывающейся поверхности, отвечающей кривой разрежения $i$-го семейства:

$$
\vec{y}=\left(\begin{array}{c}
\lambda^{i} \\
u^{1} \lambda^{i}-f^{1} \\
\cdots \cdots \\
u^{n} \lambda^{i}-f^{n}
\end{array}\right)
$$

В силу соотношения $L_{i} \lambda^{i}=0$ имеем $L_{i} \vec{y}=0$, т. е. ребро возврата вырождается в точку. Теорема доказана. 
СлЕДСТВИЕ. Для слабо нелинейных систем законов сохранения все развертыввющиеся поверхности являются коническими, а все фокальные гиперповерхности вырождаются в подмногообразия коразмерности $\geqslant 2$.

Таким образом, системам законов сохранения класса Темпля соответствуют семейства прямых, все развертываюшиеся поверхности которых являются либо плоскими, либо коническими. Поскольку оба эти свойства очевидным образом проективно инвариантны, мы получаем прозрачное геометрическое доказательство инвариантности систем класса Темпля относительно преобразований по решению (12) (см. теорему 2). Подчеркнем, что на языке систем законов сохранения факт инвариантности систем класса Темпля относительно преобразований по решению (12) был далеко не так очевиден.

Теоремы 9 и 10 позволяют дать совершенно элементарное геометрическое доказательство теоремы 1. Из определения темплевских систем, а также теорем 6 и 7 следует, что все развертывающиеся поверхности обладают следующим свойством: для любой прямолинейной образующей этой поверхности все другие прямолинейные образующие пересекают ее. Если любые три из образующих пересекаются в одной точке, то это - конические поверхности. В противном случае все образующие лежат в плоскости тех трех, которые, попарно пересекаясь, не проходят через одну точку. Учитывая теперь утверждения теорем 9 и 10, получаем теорему 1.

ПримеР 3. Системе трех уравнений (см. пример 2)

$$
\begin{aligned}
a_{t} & =b_{x}, \\
b_{t} & =c_{x}, \\
c_{t} & =\left(b^{2}-a c\right)_{x}
\end{aligned}
$$

отвечает трехпараметрическое семейство прямых

$$
\begin{aligned}
& y^{1}=a y^{0}-b, \\
& y^{2}=b y^{0}-c, \\
& y^{3}=c y^{0}-b^{2}+a c,
\end{aligned}
$$

фокальные поверхности которого задаются формулами (38):

$$
\begin{aligned}
& y^{0}=\lambda, \\
& y^{1}=a \lambda-b, \\
& y^{2}=b \lambda-c, \\
& y^{3}=c \lambda-b^{2}+a c,
\end{aligned}
$$

где $\lambda$ - корень характеристического уравнения

$$
\lambda^{3}+a \lambda^{2}-2 b \lambda+c=0
$$

Несложно показать, что все три "куска" фокальной поверхности (44), отвечающие трем различным значениям $\lambda$, на самом деле "склеены" в одно алгебраическое многообразие степени девять

$$
\begin{aligned}
y^{2}-y^{1} y^{0}-y^{0^{3}} & =0, \\
y^{3} y^{0^{2}}+y^{2^{2}} & =0 .
\end{aligned}
$$


Фокальная поверхность (45) имеет коразмерность 2 , поскольку рассматриваемая система является слабо нелинейной. Она обладает следующими двумя важными свойствами.

1. Многообразие трисекант поверхности (45) зависит от трех параметров. (Напомним, что трисекантой называется прямая, пересекаюшая поверхность в трех точках.)

2. Для фиксированной точки $p$ на поверхности (45) множество трисекант, проходяших через $p$, образует плоский пучок с вершиной в точке $p$ (следствие прямолинейности кривых разрежения).

Можно показать, что при некоторых естественных ограничениях свойства 1 и 2 определяют поверхность (45) однозначно с точностью до проективной эквивалентности.

ПРИмеР 4. Рассмотрим систему трех законов сохранения

$$
\begin{aligned}
a_{t} & =b_{x}, \\
b_{t} & =c_{x}, \\
c_{t} & =(b c / a)_{x},
\end{aligned}
$$

которая подробно исследовалась в [8]. Характеристические скорости этой системы имеют вид $\lambda=b / a, \pm \sqrt{c / a}$. В силу слабой нелинейности все куски фокальной поверхности имеют коразмерность 2 , причем фокальная поверхность, отвечаюшая характеристической скорости $\lambda=b / a$, является двумерной плоскостью

$$
y^{1}=y^{3}=0
$$

в то время как фокальные поверхности, отвечаюшие характеристическим скоростям $\lambda= \pm \sqrt{c / a}$, склеиваются в одно алгебраическое многообразие степени 4

$$
\begin{aligned}
& y^{2}+y^{1} y^{0}=0 \\
& y^{3}+y^{2} y^{0}=0
\end{aligned}
$$

Как и в предыдущем примере, плоскость (47) и поверхность (48) обладают следующими свойствами:

1. Имеется ровно трехпараметрическое семейство прямых, которые пересекают плоскость (47) и имеют две общие точки с поверхностью (48).

2. Для фиксированной точки $p$ на (47) или (48) множество прямых семейства, проходящих через $p$, образует плоский пучок с вершиной в точке $p$.

Примеры 3 и 4 приводят к следуюшей интересной задаче: с точностью до проективной эквивалентности классифицировать подмногообразия $M^{n-1} \subset A^{n+1}$ такие, что

1) множество $n$-секант подмногообразия $M^{n-1}$ зависит от $n$ параметров;

2 ) для фиксированной точки $p \in M^{n-1}$ множество $n$-секант, проходящих через $p$, образует плоский пучок с вершиной в точке $p$.

Простейшим примером такого рода является многообразие $M^{n-1}$, распадающееся в объединение $n$ плоскостей коразмерности 2 в $A^{n+1}$. 
Мы надеемся привести результаты классификации в отдельной работе. Согласно теоремам 9 и 10 сформулированная геометрическая задача эквивалентна описанию слабо нелинейных систем законов сохранения с прямолинейными кривыми разрежения, т. е. систем, которые являются "дважды" темплевскими.

Теоремы 9 и 10 позволяют дать явное описание систем из двух законов сохранения класса Темпля. Рассмотрим в отдельности системы каждого из трех типов, выделенных в $\S 3$.

I тип. Кривые разрежения обоих семейств являются прямыми.

В этом случае развертьваюшиеся поверхности, отвечаюшие кривым разрежения первого и второго семейств, представляют собой два однопараметрических семейства плоскостей. Каждое из них огибает свою полость фокальной поверхности, которая тем самым будет развертывающейся. Прямые такой конгруэнции - это линии попарных пересечений двух однопараметрических семейств плоскостей.

Параметризуем плоскости этих двух семейств параметрами $p$ и $q$ следующим образом:

$$
\begin{gathered}
g^{1}(p) y^{0}+p y^{1}-y^{2}-h^{1}(p)=0 \\
g^{2}(q) y^{0}+q y^{1}-y^{2}-h^{2}(q)=0 .
\end{gathered}
$$

Разрешая эти уравнения относительно $y^{1}, y^{2}$, мы получим уравнения соответствующего двухпараметрического семейства прямых:

$$
\begin{aligned}
& y^{1}=u^{1} y^{0}-f^{1}=\frac{g^{1}(p)-g^{2}(q)}{q-p} y^{0}-\frac{h^{1}(p)-h^{2}(q)}{q-p} \\
& y^{2}=u^{2} y^{0}-f^{2}=\frac{q g^{1}(p)-p g^{2}(q)}{q-p} y^{0}-\frac{q h^{1}(p)-p h^{2}(q)}{q-p} .
\end{aligned}
$$

Как легко видеть, полученные формулы в точности совпадают с (26).

Это рассуждение обобщается на $n \geqslant 3$ и позволяет дать полное описание систем класса Темпля с прямолинейными кривыми разрежения при дополнительном предположении о наличии инвариантов Римана. Соответствующие этим системам семейства прямых устроены следующим образом: все $n$ фокальных гиперповерхностей являются развертывающимися, т.е. огибающими однопараметрических семейств гиперплоскостей. Сами прямые семейства есть прямые пересечения $n$ однопараметрических семейств гиперплоскостей в $A^{n+1}$.

Параметризуем гиперплоскости этих семейств параметрами $R^{1}, \ldots, R^{n}$ в виде

$$
\sum_{j=1}^{n} s_{j}^{i}\left(R^{i}\right) y^{j}=R^{i} y^{0}+1, \quad i=1, \ldots, n
$$

Разрешая эти уравнения относительно $y^{1}, \ldots, y^{n}$, мы получим явные параметрические формулы для плотностей и токов диагонализуемых систем с прямолинейными кривыми разрежения, которые обобщают формулы (26). Детали этой конструкции, а также описание систем класса Темпля при $n=3$ мы предполагаем изложить в отдельной работе. 
II тип. Характеристические скорости обоих семейств являются слабо нелинейньмии.

В этом случае фокальные поверхности соответствующей конгруэнции вырождаются в кривые. Сама конгруэнщия образована прямыми, которые пересекают каждую из этих кривых. Пусть

$$
\vec{j}_{1}=\left(\begin{array}{c}
p \\
-q^{1}(p) \\
-h^{1}(p)
\end{array}\right), \quad \vec{j}_{2}=\left(\begin{array}{c}
q \\
-q^{2}(q) \\
-h^{2}(q)
\end{array}\right)
$$

есть радиус-векторы кривых. Условие того, что прямая

$$
\begin{aligned}
& y^{1}=u^{1} y^{0}-f^{1}(u), \\
& y^{2}=u^{2} y^{0}-f^{2}(u)
\end{aligned}
$$

проходит через точки $\vec{j}_{1}$ и $\vec{j}_{2}$, имеет вид

$$
\begin{aligned}
-q^{1} & =u^{1} p-f^{1}, & & -q^{2}=u^{1} q-f^{1}, \\
-h^{1} & =u^{2} p-f^{2}, & & -h^{2}=u^{2} q-f^{2} .
\end{aligned}
$$

Выражая отсюда $u^{i}, f^{i}$, мы приходим к формулам (28).

Аналогичные рассуждения позволяют получить описание систем из $n$ законов сохранения, все характеристические скорости которых являются слабо нелинейными (заметим, что условие слабой нелинейности системы (1) эквивалентно $n$ уравнениям второго порядка на функции $f^{i}$, произвол в решении которых составляет тем самым $2 n$ функций от $n-1$ аргумента). При этом фокальные гиперповерхности вырождаются в $n$ подмногообразий коразмерности 2 в $A^{n+1}$. Несложно показать, что в общем случае имеется ровно $n$-параметрическое семейство прямых, которые пересекают все эти подмногообразия. Поскольку конструкция однозначно фиксируется выбором $n$ подмногообразий коразмерности 2 в $A^{n+1}$, произвол составляет $2 n$ функций $n-1$ аргумента.

III тип. Кривые разрежения одного семейства являются прямыми, а характеристическая скорость другого семейства является слабо нелинейной.

В этом случае одно семейство развертывающихся поверхностей состоит из плоскостей. Эти плоскости огибают полость фокальной поверхности, которая должна вырождаться в кривую в силу условия слабой нелинейности. Но это значит, что сама кривая есть прямая линия. Итак, одна из фокальных поверхностей вырождается в прямую. Наша конгруэнция состоит из всех прямых, которые пересекают данную прямую и касаются данной поверхности. Направим эту прямую вдоль оси $y^{0}$. Тогда уравнения прямых, проходящих через ось $y^{0}$, имеют вид

$$
\begin{aligned}
& y^{1}=u^{1}\left(y^{0}-g\right), \\
& y^{2}=u^{2}\left(y^{0}-g\right),
\end{aligned}
$$

где функция $g\left(u^{1}, u^{2}\right)$ однозначно определяется из условия касания прямой со второй полостью фокальной поверхности. Полученные формулы в точности совпадают с (31).

Наглядность и простота приведенных выше рассуждений убедительно свидетельствуют в пользу предлагаемого нами геометрического подхода. Однако их непосредственное обобщение уже на случай систем трех законов сохранения сталкивается с определенными трудностями и выходит за рамки настоящей работы. 


\section{§7. Корреляции трехмерного проективного пространства как преобразования систем класса Темпля}

Корреляиией трехмерного проективного пространства называется такое отображение, определенное на множестве его прямых, которое каждой связке прямых сопоставляет совокупность прямых, лежащих в одной плоскости, и наоборот, совокупности прямых, лежащих в одной плоскости, сопоставляет некоторую связку прямых (см., например, [17]). Примером корреляции может служить преобразование, сопоставляющее прямой

$$
\begin{aligned}
& y^{1}=u^{1} y^{0}-f^{1}, \\
& y^{2}=u^{2} y^{0}-f^{2}
\end{aligned}
$$

прямую

$$
\begin{aligned}
& y^{1}=u^{1} y^{0}-u^{2}, \\
& y^{2}=f^{1} y^{0}-f^{2},
\end{aligned}
$$

т. е. преобразование (29):

$$
u^{1} \rightarrow u^{1}, \quad u^{2} \rightarrow f^{1}, \quad f^{1} \rightarrow u^{2}, \quad f^{2} \rightarrow f^{2} .
$$

В самом деле, при этом преобразовании связка прямых (49) с вершиной в точке $\left(\alpha^{0}, \alpha^{1}, \alpha^{2}\right)$ переходит в совокупность прямых (50), лежаших в плоскости

$$
\alpha^{1} y^{0}-\alpha^{0} y^{1}+y^{2}-\alpha^{2}=0 .
$$

Из определения корреляции автоматически вытекает, что композиция любых двух корреляций является проективным преобразованием. Поэтому произвольная корреляция может быть получена как композиция корреляции (29) и проективного преобразования.

Применяя корреляцию к конгруэнции прямых трехмерного пространства, мы получим некоторую новую конгруэнцию прямых. Корреляция переводит развертывающиеся поверхности конгруэнщии в развертываюшиеся поверхности. Действительно, кривые разрежения (а они соответствуют развертывающимся поверхностям) системы (20)

$$
\begin{aligned}
u_{t}^{1} & =f^{1}(u)_{x}, \\
u_{t}^{2} & =f^{2}(u)_{x}
\end{aligned}
$$

есть интегральные кривые векторных полей

$$
\frac{\partial}{\partial u^{1}}+\frac{\lambda^{i}-f_{1}^{1}}{f_{2}^{1}} \frac{\partial}{\partial u^{2}}
$$

(см. (22)), где $\lambda^{i}$ удовлетворяют уравнению (21):

$$
\lambda^{2}-\left(f_{1}^{1}+f_{2}^{2}\right) \lambda+\left(f_{1}^{1} f_{2}^{2}-f_{2}^{1} f_{1}^{2}\right)=0 .
$$


После замены (29)

$$
u^{1} \rightarrow u^{1}, \quad u^{2} \rightarrow f^{1}, \quad f^{1} \rightarrow u^{2}, \quad f^{2} \rightarrow f^{2}
$$

система переходит в

$$
\begin{aligned}
u_{t}^{1} & =u_{x}^{2}, \\
f^{1}(u)_{t} & =f^{2}(u)_{x} .
\end{aligned}
$$

Ее характеристические векторы можно выбрать в виде

$$
\frac{\partial}{\partial u^{1}}+\mu^{i} \frac{\partial}{\partial u^{2}}
$$

где $\mu^{i}$ удовлетворяют уравнению

$$
\mu^{2}-\left(\frac{f_{2}^{2}-f_{1}^{1}}{f_{2}^{1}}\right) \mu-\frac{f_{1}^{2}}{f_{2}^{1}}=0
$$

Используя формулы Виета, легко показать, что $\mu^{i}=\frac{\lambda^{i}-f_{1}^{1}}{f_{2}^{1}}$.

Таким образом, характеристические векторы систем совпадают, а значит, развертываюшиеся поверхности при корреляции переходят в развертываюшиеся поверхности. При этом конические развертываюшиеся поверхности становятся плоскими, и наоборот, плоские - коническими. Следовательно, конгруэнции, отвечающие системам класса Темпля типа I, перейдут в конгруэнции, отвечающие системам класса Темпля типа II. Ударные адиабаты систем, связанных преобразованием (29), задаются одним и тем же уравнением

$$
\left(u^{1}-u_{0}^{1}\right)\left(f^{2}-f_{0}^{2}\right)=\left(u^{2}-u_{0}^{2}\right)\left(f^{1}-f_{0}^{1}\right)
$$

т. е. корреляция сохраняет и ударную адиабату.

Это дает дифференциально-геометрическую интерпретацию преобразования (29), связывающего системы (25) и (27) (см. §3).

\section{§8. Заключение}

В работе предложен новый подход к системам законов сохранения, позволяющий переформулировать многие возникаюшие здесь понятия и конструкции на геометрическом языке теории конгруэнций.

Следуюшие задачи представляются нам наиболее интересньми для дальнейшего исследования.

1. Дать описание систем $n$ законов сохранения класса Темпля, или же, что эквивалентно, $n$-параметрических семейств прямых в $A^{n+1}$, все развертываюшиеся поверхности которых являются либо плоскими, либо коническими. (Приведенная в $\S 5$ простая и чисто геометрическая классификация систем из двух законов сохранения класса Темпля позволяет надеяться на эффективное решение этой задачи и в более сложном случае $n \geqslant 3$.)

2. Изучить системы законов сохранения, отвечающие тем или иным проективно инвариантным классам $n$-параметрических семейств прямых в $A^{n+1}$. (Для $n=2$ 
это есть не что иное, как перевод “теории конгруэнций” [14] на язык систем законов сохранения.)

3. Изучить последовательности конгруэнщий и отвечающих им систем законов сохранения, порожденных преобразованиями Лапласа (см. [20]).

Работа одного из авторов, Е.В. Ферапонтова, выполнена при частичной финансовой поддержке РФФИ, гранты № 96-01-00166, 96-01-01297, 96-06-80104a и INTAS. Работа другого автора, С.И. Агафонова, - при финансовой поддержке Тверьуниверсалбанка.

\section{Приложение}

В таблице не указаны переменные, которые не преобразуются.

\begin{tabular}{|c|c|c|c|}
\hline $\begin{array}{c}\text { Опера- } \\
\text { тор }\end{array}$ & \begin{tabular}{|c|} 
Конечное \\
преобразование
\end{tabular} & $\begin{array}{c}\text { Замена перемен- } \\
\text { ных системы }\end{array}$ & $\begin{array}{c}\text { Проективное } \\
\text { преобразование }\end{array}$ \\
\hline$T U_{1}$ & $\tilde{u}^{1}=u^{1}+\epsilon$ & $\tilde{u}^{1}=u^{1}+\epsilon$ & $\tilde{y}^{1}=y^{1}+\epsilon y^{0}$ \\
\hline$T U_{2}$ & $\tilde{u}^{2}=u^{2}+\epsilon$ & $\tilde{u}^{2}=u^{2}+\epsilon$ & $\tilde{y}^{2}=y^{2}+\epsilon y^{0}$ \\
\hline$T F_{1}$ & $\tilde{f}^{1}=f^{1}+\epsilon$ & $\tilde{f}^{1}=f^{1}+\epsilon$ & $\tilde{y}^{1}=y^{1}-\epsilon$ \\
\hline$T F_{2}$ & $\tilde{f}^{2}=f^{2}+\epsilon$ & $\tilde{f}^{2}=f^{2}+\epsilon$ & $\tilde{y}^{2}=y^{2}-\epsilon$ \\
\hline$D_{0}$ & $\begin{array}{l}\tilde{u}^{1}=u^{1} e^{\epsilon} \\
\tilde{u}^{2}=u^{2} e^{\epsilon}\end{array}$ & $d T=e^{\epsilon} d t$ & $\tilde{y}^{0}=e^{-\epsilon} y^{0}$ \\
\hline$D_{1}$ & $\begin{array}{l}\tilde{f}^{1}=f^{1} e^{\epsilon} \\
\tilde{f}^{2}=f^{2} e^{\epsilon}\end{array}$ & $d X=e^{\epsilon} d x$ & $\begin{array}{l}\tilde{y}^{0}=e^{\epsilon} y^{0} \\
\tilde{y}^{1}=e^{\epsilon} y^{1} \\
\tilde{y}^{2}=e^{\epsilon} y^{2}\end{array}$ \\
\hline$D_{2}$ & $\begin{array}{l}\tilde{u}^{2}=u^{2} e^{\epsilon} \\
\tilde{f}^{2}=f^{2} e^{\epsilon}\end{array}$ & $\tilde{u}^{2}=u^{2} e^{\epsilon}$ & $\tilde{y}^{2}=e^{\epsilon} y^{2}$ \\
\hline$R_{1}$ & $\begin{array}{l}\tilde{u}^{1}=u^{1}+\epsilon f^{1} \\
\tilde{u}^{2}=u^{2}+\epsilon f^{2}\end{array}$ & $d T=d t-\epsilon d x$ & $\begin{aligned} \tilde{y}^{0} & =\frac{y^{0}}{1+\epsilon y^{0}} \\
\tilde{y}^{1} & =\frac{y^{1}}{1+\epsilon y^{0}} \\
\tilde{y}^{2} & =\frac{y^{2}}{1+\epsilon y^{0}}\end{aligned}$ \\
\hline$R_{2}$ & $\begin{array}{l}\tilde{f}^{1}=f^{1}+\epsilon u^{1} \\
\tilde{f}^{2}=f^{2}+\epsilon u^{2}\end{array}$ & $d X=d x-\epsilon d t$ & $\tilde{y}^{0}=y^{0}+\epsilon$ \\
\hline
\end{tabular}




\begin{tabular}{|c|c|c|c|}
\hline $\begin{array}{c}\text { Oпера- } \\
\text { тор }\end{array}$ & $\begin{array}{c}\text { Конечное } \\
\text { преобразование }\end{array}$ & $\begin{array}{c}\text { Замена перемен- } \\
\text { ных системы }\end{array}$ & $\begin{array}{c}\text { Проективное } \\
\text { преобразование }\end{array}$ \\
\hline$R_{1,2}$ & $\tilde{u}^{1}=u^{1}+\epsilon u^{2}$ & $\tilde{u}^{1}=u^{1}+\epsilon u^{2}$ & $\tilde{y}^{1}=y^{1}+\epsilon y^{2}$ \\
& $\tilde{f}^{1}=f^{1}+\epsilon f^{2}$ & & \\
\hline$R_{2,1}$ & $\tilde{u}^{2}=u^{2}+\epsilon u^{1}$ & $\tilde{u}^{2}=u^{2}+\epsilon u^{1}$ & $\tilde{y}^{2}=y^{2}+\epsilon y^{1}$ \\
& $\tilde{f}^{2}=f^{2}+\epsilon f^{1}$ & & \\
\hline$P U_{1}$ & $\tilde{u}^{1}=\frac{u^{1}}{1-\epsilon u^{1}}, \quad \tilde{f}^{1}=\frac{f^{1}}{1-\epsilon u^{1}}$ & $d X=\left(1-\epsilon u^{1}\right) d x$ & $\tilde{y}^{0}=y^{0}-\epsilon y^{1}$ \\
& $\tilde{u}^{2}=\frac{u^{2}}{1-\epsilon u^{1}}, \quad \tilde{f}^{2}=f^{2}+\frac{\epsilon u^{2} f^{1}}{1-\epsilon u^{1}}$ & $-\epsilon f^{1} d t$ & \\
\hline$P U_{2}$ & $\tilde{u}^{1}=\frac{u^{1}}{1-\epsilon u^{2}}, \quad \tilde{f}^{1}=f^{1}+\frac{\epsilon u^{1} f^{2}}{1-\epsilon u^{2}}$ & $d X=\left(1-\epsilon u^{2}\right) d x$ & $\tilde{y}^{0}=y^{0}-\epsilon y^{2}$ \\
& $\tilde{u}^{2}=\frac{u^{2}}{1-\epsilon u^{2}}, \quad \tilde{f}^{2}=\frac{f^{2}}{1-\epsilon u^{2}}$ & $-\epsilon f^{2} d t$ & \\
\hline$P F_{1}$ & $\tilde{u}^{1}=\frac{u^{1}}{1-\epsilon f^{1}}, \quad \tilde{f}^{1}=\frac{f^{1}}{1-\epsilon f^{1}}$ & $d T=-\epsilon u^{1} d x$ & $\tilde{y}^{0}=\frac{y^{0}}{1+\epsilon y^{1}}$ \\
& $\tilde{u}^{2}=u^{2}+\frac{\epsilon u^{1} f^{2}}{1-\epsilon f^{1}}, \quad \tilde{f}^{2}=\frac{f_{2}}{1-\epsilon f^{1}}$ & $+\left(1-\epsilon f^{1}\right) d t$ & $\tilde{y}^{1}=\frac{y^{1}}{1+\epsilon y^{1}}$, \\
& & & $\tilde{y}^{2}=\frac{y^{2}}{1+\epsilon y^{1}}$ \\
\hline$P F_{2}$ & $\tilde{u}^{1}=u^{1}+\frac{\epsilon u^{2} f^{1}}{1-\epsilon f^{2}}, \quad \tilde{f}^{1}=\frac{f^{1}}{1-\epsilon f^{2}}$ & $d T=-\epsilon u^{2} d x$ & $\tilde{y}^{0}=\frac{y^{0}}{1+\epsilon y^{2}}$, \\
& $\tilde{u}^{2}=\frac{u^{2}}{1-\epsilon f^{2}}, \quad \tilde{f}^{2}=\frac{f_{2}}{1-\epsilon f^{2}}$ & $+\left(1-\epsilon f^{2}\right) d t$ & $\tilde{y}^{1}=\frac{y^{1}}{1+\epsilon y^{2}}$, \\
& & & $\tilde{y}^{2}=\frac{y^{2}}{1+\epsilon y^{2}}$ \\
\hline
\end{tabular}

\section{Список литературы}

1. Рождественский В.Л., Яненко Н.Н. Системы квазилинейных уравнений и их приложения к газовой динамике. М.: Наука, 1968.

2. Lax P.O. Hyperbolic systems of conservation laws. II // Comm. in Pure and Appl. Math. 1957. V. 10. P. 537-566.

3. Temple B. Systems of conservations laws with invariant submanifolds // Transactions of the American Mathematical Society. 1983. V. 280. № 2. P. 781-795.

4. Alekseevskaya T.V. Study of the system of quasilinear equations for isotachophoresis // Adv. in Appl. Math. 1990. V. 11. № 1. P. 63-107.

5. Dubrovin B.A. Geometry of $2 D$ topological field theories. Preprint SISSA-89/94/FM, SISSA, Trieste, 1994. hep-th/9407018.

6. Mokhov O.I. Symplectic and Poisson geometry on loop spaces of manifolds and nonlinear equations // Trans. Amer. Math. Soc. Ser. 2. 1995. V. 170. P. 121-151.

7. Мохов О.И., Ферапонтов Е.В. Уравнения ассоциативности двумерной топологической теории поля как интегрируемые гамильтоновы системы гидродинамического типа // Функц. анализ и его прилож. 1996. Т. 30. № 3. С. 62-72.

8. Ferapontov E.V., Mokhov O.I. The equations of associativity as hydrodynamic type systems: Hamiltonian representation and the integrability. In Proc. of the workshop "Nonlinear Physics. Theory and experiment". Italy. Lecce: World Scientific Singapore, 1995.

9. Rogers C., Shadwick W.F. Backlund transformations and their Applications. N.Y.: Academic Press, 1982.

10. Rogers C. Reciprocal transformations and their Applications. Nonlinear Evolutions // Proc. of the 5th Workshop on Nonlinear Evolution Equations. France, 1987. P. 109-123.

11. Ферапонтов Е.В. Преобразования по решению и их инварианты // Дифференц. уравн. 1989. T. 25. № 7. C. $1256-1265$. 
12. Ферапонтов E.B. Автопреобразования по решению и гидродинамические симметрии // Дифференц. уравн. 1991. Т. 27. № 7. С. 1250-1262.

13. Ferapontov E. V. Dupin hypersurfaces and integrable hamiltonian systems of hydrodynamic type, which do not possess Riemann invariants // Diff. Geometry and its Appl. 1995. V. 5. P. 121-152.

14. Фиников С.П. Теория конгруэнций. М.- Л.: Гостехиздат, 1950.

15. Царев С.П. Геометрия гамильтоновых систем гидродинамического типа. Обобщенный метод годограффа // Изв. АН СССР. Сер. матем. 1990. Т. 54. № 5. С. 1048-1068.

16. Ферапонтов E.B. Гамильтоновы системы гидродинамического типа и их реализация на гиперповерхностях псевдоевклидова пространства // ВИНИТИ. Проблемы геометрии. 1990. T. 22. C. 59-96.

17. Розенфельд Б.А. Многомерные пространства. М.: Физматлит, 1966.

18. Little J.B. On Lie's approach to the study of translation manifolds // J. Diff. Geom. 1987. V. 26. № 2. P. 253-272.

19. Lie S. Translations - $M_{3}$ zweiter art im $R_{4}$. Gesammelte Abhandlungen, Band VII, Abh. XXIX, Teubner, Leipzig, 1960.

20. Ферапонтов Е.В. Преобразования Лапласа систем гидродинамического типа в инвариантах Римана. (Принята к печати в журн. “Теор. и мат. физ.”, 1997.)

Поступило в редакцию

29.IV.1996 